Voix et Images

\title{
Une fragile renaissance : images du corps masculin dans les Masques et le Passager
}

\section{Agnès Whitfield}

Volume 15, numéro 3 (45), printemps 1990

Gilbert La Rocque

URI : https://id.erudit.org/iderudit/200855ar

DOI : https://doi.org/10.7202/200855ar

Aller au sommaire du numéro

Éditeur(s)

Université du Québec à Montréal

ISSN

0318-9201 (imprimé)

1705-933X (numérique)

Découvrir la revue

Citer cet article

Whitfield, A. (1990). Une fragile renaissance : images du corps masculin dans les Masques et le Passager. Voix et Images, 15(3), 374-386.

https://doi.org/10.7202/200855ar d'utilisation que vous pouvez consulter en ligne.

https://apropos.erudit.org/fr/usagers/politique-dutilisation/ 


\section{Une fragile renaissance: images du corps masculin dans les Masques et le Passager}

\section{par Agnès Whitfield, université Queen's}

Les Masques et le Passager: voici que le corps s'inscrit déjà dans le titre même des deux derniers romans ${ }^{1}$ de Gilbert La Rocque, mais discrètement, objet aussi banal au fond que les métaphores qui le portent. Du corps réduit à ses masques, il ne reste, dans un cas, que la peau qui cache ou qui exhibe l'identité du sujet. Dans l'autre, l'être tout entier, le corps de l'homme en l'occurrence, est livré à sa trajectoire, saisi dans ce qu'il garde de constant dans sa mouvance, sa matérialité ou seulement son volume. Simple jeu du hasard, reflet de l'ubiquité des images corporelles dans la langue ${ }^{2}$ ? Non, car les textes que coiffent ces titres donnent effectivement à voir toute une série de métaphores et d'images du corps humain, celui de la femme et de l'enfant comme celui de l'homme, corps multiples donc que l'écriture se plaît à saisir dans toutes leurs dimensions temporelles, spatiales, matérielles, fantasmatiques, sexuelles et affectives, ou bien à projeter, par métonymie, sur le monde extérieur, perçu à leur image.

Paradoxalement, si la critique souligne souvent la violence de l'écriture larocquienne ${ }^{3}$, sa fixation anale ${ }^{4}$, son obsession pour les odeurs et la putréfaction du corps ${ }^{5}$ et, surtout, l'importance accordée aux sensations fortes ${ }^{6}$, au subconscient ${ }^{7}$ et à la sexualité ${ }^{8}$, elle n'a jamais tenté de faire la synthèse de tous ces fragments corporels, de retrouver l'image globale du corps qui les sous-tend. Sans doute le sujet, à plus d'un titre d'ailleurs, manquait-il d'actualité critique

1 On pourrait en dire autant, d'ailleurs, de presque tous les titres de La Rocque.

2 Voir, à ce sujet, Pierre Guiraud, le Langage du corps, Paris, Presses universitaires de France, 1980.

3 Donald Smith, Gilbert La Rocque. L'écriture du rêve, Montréal, Québec/ Amérique, 1985, p. 39.

4 André Vanasse, "La fête, la haine, la mort ", dans Donald Smith, op. cit., p. 104.

5 Ibid.

6 Donald Smith, op. cit., p. 39.

7 Donald Smith, "Gilbert La Rocque ou comment l'écrivain se fait l'interprète de son subconscient", l'Écrivain devant son œuvre. Entrevues, Montréal, Québec/Amérique, 1983, p. 293-312.

8 Alain Piette, «Focalisation, voyeurisme et scène originaire dans Serge d'entre les morts ", Voix \& images, $n^{\circ} 36$, printemps 1987 , p. 497-511. 
(l'Index-Thesaurus 1967-1987 de Voix \& images ne cite que deux articles portant sur le thème du corps ${ }^{9}$ ) ainsi que de statut théorique propice. Pendant longtemps, en effet, la notion du corps, comme dimension vitale de la réalité humaine, comme donnée globale présexuelle et irréductible ${ }^{10}$ a constitué le grand refoulé des théories psychanalytiques de Freud et de Lacan dont s'inspirent la majorité des études larocquiennes.

L'ouverture actuelle de la problématique du corps, dans le cadre notamment du féminisme ${ }^{11}$, à ses dimensions culturelles, nous oblige pourtant à reposer la question de sa représentation dans l'écriture larocquienne. Aussi ne s'agit-il plus de ramener le corps aux complexes ponctuels qui le parcourent, mais de le considérer, dans son ensemble, comme une construction culturelle susceptible d'éclairer, par-delà sa force ou sa fragilité, son intégrité ou sa fragmentation, l'identité du sujet qui l'habite et la place de celui-ci dans la culture. Sujet masculin ou sujet féminin? La perspective narrative des Masques et du Passager, axée en général sur le discours intérieur de personnages masculins, ainsi que la prédominance des métaphores et des images touchant au corps de l'homme et de l'enfant mâle nous amènent ici à privilégier la représentation du corps masculin. Mais ce choix s'impose aussi pour une autre raison. Contrairement au corps féminin dont les divers codes de représentation ont été maintenant en grande partie déconstruits, le corps masculin, pour peu que l'on tente de le sortir du carcan du patriarcat ${ }^{12}$, demeure encore dans l'ombre, du côté de celui qui voit mais qui n'est pas vu. Paradoxale ironie du sort que cet effacement, alors que, rendue doublement problématique par l'éclatement tant de la Loi du Père que du sujet "moderne", la représentation du corps masculin est de plus en plus appelée, de gré ou de force, à se transformer, à se renouveler.

Deux critiques n'en soulèvent pas moins l'envers et l'endroit de cette question dans le contexte québécois ${ }^{13}$. Partant d'une réflexion

9 Pierre Hébert et Bill Winder, Index-Thesaurus 1967-1987, Montréal, Université du Québec à Montréal, 1987, p. 182. Il s'agit de deux études portant sur l'œuvre de Monique Bosco et de Yolande Villemaire. On pourrait citer aussi à ce propos le numéro spécial de Voix \& images consacré à Roger Des Roches, malgré l'ambivalence du titre ("Le propre du corps. Roger Des Roches", Voix \& images, $n^{\circ} 38$, hiver 1988).

10 D. Anzieu, "Le Moi-peau», Nouvelle Revue de psychanalyse, Paris, Gallimard, 1974, p. 194.

11 Voir Patricia Smart, Écrire dans la maison du père, Montréal, Québec/ Amérique, 1989.

12 Voir a ce sujet M. Kaufman, Beyond Patriarchy: essays by men on pleasure, power and changes (ouvrage collectif), Toronto, Oxford University Press, 1987.

13 A ce sujet, Lucie Robert remarque quelques exemples d'une nouvelle dramaturgie masculine qui fait face au féminisme comme pour relever un défi, comme pour se re-définir, se re-situer («De l'epique à l'intime: regard sur la dramaturgie masculine», Voix \& images, $n^{\circ} 33$, printemps 1986, p. 579). 
sur la modernité au Québec, Pierre Nepveu souligne l'importance, chez des poètes comme Paul-Marie Lapointe, Gaston Miron, Gilles Hénault, Fernand Ouellette et Jacques Brault, de la quête d'une identification paternelle ${ }^{14}$ : Redonner vie au père, être soi-même des pères: l'essor moderniste des années soixante [...] fondé sur le pouvoir créateur du moi, devait passer par là.$^{15} \mathrm{Si}$ Nepveu ne relie pas explicitement cette quête à la réinvention du corps paternel et filial, la dimension corporelle ressort néanmoins clairement du passage de "Mémoire" de Jacques Brault qu'il cite à l'appui de son hypothèse:

Cela vient de loin cela qui est mon corps

vêtu de honte et de nouveauté

comme cet homme rompu de misère

et que j'accueille dans ma haine

cet homme mon père et en qui je suis le père. ${ }^{16}$

De même, le corps sous-tend implicitement le parcours inévitablement tragique des fils découvrant leur solitude [...]. Solitude qui est celle de l'ordre paternel lui-même, du moi conquérant, héroïque, mais par là à jamais orphelin, à jamais incapable, malgré toutes ses prétentions, d'embrasser le réel à bras le corps ${ }^{17}$.

Ce rapport entre le corps et le sujet masculin dans la littérature québécoise contemporaine est abordé plus explicitement par Patricia Smart dans son essai provocateur, Écrire dans la maison du père ${ }^{18}$. À ses yeux, ce qui rend ce rapport si problématique, c'est la difficulté qu'éprouve le sujet masculin à réconcilier son désir de maîtrise avec une revalorisation du corps féminin, c'est-à-dire maternel:

Dans l'écriture des romanciers masculins, l'émergence du corps et de tout le domaine "sémiotique" ou "maternel» [...] se heurtait au désir de maîtrise du sujet masculin, produisant une écriture éclatée - mais "négativement" éclatée, si l'on peut dire, à l'image d'une forteresse qui serait assaillie de l'extérieur. 19

Selon l'optique féministe de Smart, l'origine de la solitude tragique que souligne Nepveu se situerait dans la circularité du trajet du sujet masculin emprisonné dans la Maison du père ${ }^{20}$. À cet égard, les œuvres d'Hubert Aquin et de Réjean Ducharme offriraient une écriture de transition, consciente du dilemme mais incapable de le

14 Pierre Nepveu, l'Écologie du réel, Montréal, Boréal, 1988, p. 72.

15 Ibid.

16 Jacques Brault, Poèmes 1, Saint-Lambert, le Noroit, 1986, p. 82.

17 Pierre Nepveu, op. cit., p. 73.

18 Voir aussi Patricia Smart, "Impasses ou issues? Limaginaire masculin face à la femme", Voix $\&$ images, $n^{\circ} 36$, printemps 1987 , p. 555-560.

19 Patricia Smart, op. cit., p. 255.

20 Ibid., p. 252. 
transcender. Condamné par l'idéologie dualiste du patriarcat à "vivre dans sa tête", affirme Smart, il semble que le fils ne peut chercher issue que là, dans la lucidité hyperconsciente de son écriture. ${ }^{21}$

Ambivalent à l'égard du féminisme ${ }^{22}$, sartrien, selon Donald Smith, par sa philosophie de la lucidité ${ }^{23}$, mais doté du don admirable, aux dires de Gérard Bessette, de se substituer à ses personnages féminins de les sentir cénesthésiquement ${ }^{24}$, Gilbert La Rocque appartiendrait-il à son tour à cette écriture de transition ou parvientil plutôt à re-définir le sujet masculin? Interroger les images du corps de l'homme dans les Masques et le Passager, c'est donc déborder l'analyse de l'œuvre de La Rocque proprement dite pour situer celleci, du moins dans sa phase ultime, dans le contexte plus large de la représentation problématique du corps et du sujet masculins par les écrivains québécois contemporains.

À première vue, les Masques et le Passager nous présentent en effet l'image négative d'un corps rejeté, éclaté, saisi essentiellement par ses parties: la tête, les lèvres, les tempes, les mains, les pieds, le ventre, la poitrine, l'organe sexuel et la peau. Cette fragmentation est particulièrement frappante lorsque le narrateur regarde le corps des autres, tel Alain dans les Masques qui s'imagine le réveil des Montréalais: cela montrerait des bedaines molles de buveurs de bière, des peaux mièvres, des grasseurs de torses à petits poils, des pantalons ballants, des enfourchures et des pantoufles douteuses ${ }^{25}$; tel Bernard dans le Passager qui observe la foule réunie à une réception mondaine:

il voyait des cernes de transpiration dans des dos de chemises, sous des aisselles, dos reluisants de ces médèmes, fronts. dégoulinants de ces messieurs, ça soufflait court, se pressait, macérait dans des sous-vêtements déjà trempés, le dessous des pieds flicflaquant dans les souliers [.... ${ }^{26}$

Lorsque le sujet masculin se perçoit lui-même, pourtant, la désarticulation corporelle s'atténue pour prendre surtout la forme d'une scission entre le corps et l'esprit: sa tête seule se rendait à l'évidence et le croyait, comme si elle avait été détachée de son corps (M, p. 167); cela [...] lui passait dans la tête, tandis qu'il se balançait légèrement sur

21 Ibid.

22 L'attitude de La Rocque envers la critique féministe est une des questions soulevées par Donald Smith dans son entrevue avec l'auteur (l'Écrivain devant son œuvre, op. cit., p. 307-308). André Vanasse soulève aussi la question ("La femme à la bouche rouge ", Lettres québécoises, $n^{\circ} 22$, été $1981, p .23$ ).

23. Donald Smith, Gilbert La Rocque. L'écriture du rêve, op. cit., p. 18.

24 Gérard Bessette, le Semestre, Montréal, Québec/Amérique, 1979, p. 80.

25 Gilbert La Rocque, les Masques [désormais M], Montréal, Québec/Amérique, 1980 , p. 107. Toute citation subséquente de ce texte renvoie à cette édition.

26 Gilbert La Rocque, le Passager [désormais P], Montréal, Québec/Amérique, 1984, p. 45. Toute citation subséquente de ce livre renvoie à cette édition. 
ses jambes [...] Il lui semblait qu'il était en quelque sorte coupé ou divisé en deux (P, p. 43-44).

Cette perception dualiste du sujet, conforme à l'idéologie du patriarcat, qui oppose la raison à la matière, comme la tête au corps et le masculin au féminin, sous-tend également la prolifération des images et des métaphores relatives à la tête. Effectivement, les deux protagonistes principaux des Masques et du Passager vivent beaucoup dans leur tête, pour reprendre l'expression de Smart. Nombreuses sont les formules du genre: voyant tout dans sa tête (M, p. 91); des filaments de pensées [...] papillotaient dans sa tête (P, p. 144); un torrent d'images qui [...] déboulaient dans son crâne (P, p. 72); le monde entier s'enflait dans sa tête où battait on aurait dit un moteur de paquebot (P, p. 156). Débordant d'images se télescopant [...] et s'accumulant là pour toujours (M, p. 17), la tête se transforme en véritable cinéma intérieur (M, p. 31): toute ma vie vécue pouvait presque sur commande s'étaler sous mes yeux comme une immense toile de fond où je me sentais durer, ressemblant d'une certaine façon à un cyclorama perpétuellement en mouvement (M, p. 82-83); cela lui traversait la tête comme des décharges électriques, ou comme de la pellicule de cinéma, film atroce [...] sans pour autant que cela, cette projection débridée d'images, puisse s'appeler penser (P, p. 194-195).

On sent bien que face à ce débordement, qui est en fait plutôt une implosion d'images vécues et donc sensorielles, le sujet masculin, se sentant menacé dans sa rationalité, éprouve une perte de contrôle, de maîtrise. Ainsi, assailli tant par ses souvenirs émotifs que par ses fonctions corporelles, le personnage-romancier des Masques finit-il par les confondre: tandis que, assis sur le bol de toilettes, dans l'odeur grasse et marécageuse qui montait autour de lui, il essayait de reprendre en main le contrôle de ce qui s'organisait en lui, il n'était rien d'autre qu'un effet de sa création (M, p. 36-37). Submergé par l'intensité de ses sentiments de colère, le héros du Passager éprouve une impuissance encore plus intense:

il ne parvenait plus à mettre les mots sur ce qu'il ressentait [...] des sentiments qui sortaient des profondeurs comme des points noirs, comme des fourmis, ça l'envahissait et c'était effrayant [...] c'était brusquement l'avalanche, la déboulade d'images qui papillotaient et clignotaient dans la plus complète anarchie, et cela l'écrasait véritablement, il se voyait soudain minuscule, infime, poussière à jamais anonyme et perdue dans le cosmos. (P, p. 72)

Inversement, le dégagement ou la neutralisation du corps peut renverser ce processus de décrochage verbal et cosmique pour déclencher un retour à la maîtrise: je savais bien ce que je faisais, je me sentais solide et sûr de moi, la tête dégagée, lucide, capable de faire face à la situation (M, p. 182. Nous soulignons). 
Sans doute faudrait-il relier également à ce sentiment de perte de maîtrise le penchant larocquien pour les métaphores mécaniques, ou électroniques, du corps, dont l'image d'un cinéma ou projecteur intérieur (P, p. 82) constituerait déjà un exemple. Aussi le personnage principal des Masques a-t-il l'impression que sa machine à mémoire cafouill[e] pour éviter de surcharger [s]es fusibles (M, p. 169), qu'il a une sorte de rouille dans [s]es rouages internes $(\mathbf{M}, \mathrm{p} .190)$. Le héros du Passager souffre du fait que son enveloppe hoqueteuse le transport [e] [...] sans que les centres de contrôle puissent agir efficacement sur les mouvements de son corps (P, p. 70). En panne mentale, le grippage de ses rouages intimes [...] ne tourna[nt] plus qu'avec peine et à grands grincements (P, p. 120-121), il se sent brisé, irréparable [...] dans sa tête où ça brassait tout lousse comme dans un vieux jouet mécanique dont les ressorts ont pété (P, p. 149).

Or, certainement frappantes, autant par leur fréquence que par leur conformité au dualisme patriarcal, de telles images d'un sujet masculin scindé en deux, ne parvenant plus dans sa tête hypertrophiée à contrôler une affectivité et un corps débordants, n'épuisent nullement toutes les configurations corporelles que nous offrent les Masques et le Passager. Nombreux, également, sont les passages où l'allusion explicite à l'opposition dualiste entre le corps et l'esprit véhicule en fait une certaine valorisation inverse. Le personnage-romancier des Masques souhaite souvent ${ }^{27}$ avoir une mémoire intégrale des événements à la fois cérébrale et sensorielle, qui permettrait de croire:

que ça vous est bien arrivé à vous, ailleurs que dans votre tête (que votre cœur battait pour vrai et que vos yeux ont vu tout ce que vous dites avoir vu et que vos oreilles ont entendu toutes les musiques et toutes les voix encore si vivantes en vous [...] que votre corps a été autre chose qu'un corps étranger. (M, p. 21)

De même, le roman qu'il porte en lui a besoin de fermenter encore, de se malaxer dans sa tête et dans son coeur (M, p. 20). Dans le Passager, les souvenirs du héros lui explos[ent] dans le cœur et dans la tête (P, p. 169), double lieu de sa vie intérieure (P, p. 172), de ces images issues des forces vives de son cour $(\mathbf{P}, \mathbf{p}$. 59). Il arrive également que l'esprit s'oppose ou s'ajoute à l'âme, que le héros se secoue l'esprit et l'âme (P, p. 142), ou que l'écriture reprenne plutôt le couple âme/corps: Malgré lui, il haussa les épaules comme si son corps avait voulu rassurer son âme (P, p. 144); comment savoir, comment raisonner juste dans la fureur et la honte, avec [...] une révulsion de l'âme, une grimace de tout l'intérieur $(\mathbf{P}, \mathbf{p} .105)$. Utilisés ici selon leur valeur symbolique usuelle, le cour et l'âme introduisent un terme intermédiaire dans la dichotomie corps/esprit qui permet de revaloriser l'affectivité, le cœur déplaçant le centre de la 
personnalité de l'intellectualité à l'affectivité ${ }^{28}$ et l'âme recoupant le conscient et l'inconscient dans une certaine recherche d'intégrité ${ }^{29}$.

Cette revalorisation de l'affectif, fondée néanmoins sur une image encore assez désincarnée du corps, aboutit-elle à une véritable réintégration du vécu corporel? L'utilisation des métaphores animales dans les Masques et le Passager semble en effet confirmer cette tentative d'affaiblir, sinon de dépasser, la dichotomie entre l'esprit et la chair. Certes, on trouve quelques passages où la tête s'oppose à la bestialité du corps: J'avais un peu l'impression que je ne m'habitais plus, que le locataire de ma tête avait déménagé et qu'il n'y restait plus que des crottes de rats et des reliefs sordides (M, p. 107); il ne vit pas encore ces rats qui lui rongeaient l'anus et les couilles (M, p. 74). La sexualité déclenche inévitablement des images animales, tantôt vagues (On sentait l'animal et tout râlants [...] on se lâchait lousses [...] dans le flot écumant des lubrifications [M, p. 86]), tantôt plus précises (jolis fonds de culottes de dentelles tout imprégnés de moiteurs d'organes dont la senteur vous transforme subito en chien museau flairant dans les sécrétions des fentes embrasées des chiennes [P, p. 89]). Enfin, la réalité animale de l'homme est parfois dévalorisée, mais d'une façon plutôt abstraite, le personnage masculin ouvrant animalesquement la bouche (P, p. 67) pour vomir ou bien cherchant à passer à travers l'étape animale du chagrin (M, p. 190), à fuir quelque chose dont il avait bestialement besoin de se débarrasser $(\mathrm{P}, \mathrm{p} .142)$.

Pour la plupart, pourtant, les métaphores animales engagent le corps entier dans ce qu'il comporte de vulnérable ou de blessé. Dans sa déroute physique et affective, le personnage masculin est comparé à divers animaux: un chien malade (P, p. 74), une bêté blessée (M, p. 169), un animal crevé au pied d'un arbre (M, p. 131), une souris blanche, folle blanche avec ses yeux rouges comme des. morceaux de vitre, qui tourne jusqu'à la mort dans sa cage (M, p. 74), un bœuf étique et poussiéreux $(P, p .44)$ ou une hyène qui se dévore $(P, p .145)$. Il a une envie folle de se coucher par terre comme une bête qui va mourir (P, p. 183), se secou[e] l'esprit et l'âme comme un chevreuil blessé (P, p. 142). Quelques rares métaphores offrent néanmoins une vision plus positive de l'animalité de l'homme, en consacrant son adresse et sa puissance (il bondit, comme un tigre cette fois, pour vrai, avec toute la force de son corps, dans une longue détente silencieuse $[\mathbf{P}, \mathbf{p} .118]$ ) ou en prévoyant sa réconciliation avec la sphère animale dans un futur lointain:

plus loin dans l'autre direction que les ères floues et tonnantes [...:] où grouillait un bestiaire qu'on aurait dit sorti tout droit

28 J. Chevalier et A. Gheerbrant, Dictionnaire des symboles, Paris, Robert Laffont/Jupiter, 1982, p. 263.

29 Ibid., p. 34. Combinaison des principes mâle et femelle, la symbolique de l'âme permet aussi une certaine intégration du féminin et du masculin (Ibid., p. 613). 
d'un cauchemar de la terre, des créatures inouies défiant la raison et presque les lois de la nature [...] gigantesques brouteurs de frondaison, diplodocus et brontosaures [...] l'archivieux temps des prédateurs à triples mâchoires d'acier [...] Ce serait en tout cas l'époque où il aurait enfin découvert les seules questions qu'il fallait poser, pas même les réponses, au fait, non, rien que les interrogations primordiales. (M, p. 74)

S'ajoutant donc à la revalorisation de l'affectivité opérée par les schèmes corps/âme/cœur, cette ébauche d'une image positive de redécouverte de soi, fondée sur un retour aux origines animales et matérielles de l'homme, laisse entrevoir la possibilité du moins d'une nouvelle représentation, plus intégrale, du corps. Possibilité que suggèrent aussi le caractère plutôt abstrait des métaphores animales dévalorisantes et l'absence relative des métaphores sadiques ou menaçantes qui pullulent dans des textes misogynes plus conförmes à l'idéologie dualiste ${ }^{30}$. Si les images corporelles et animales restent néanmoins contradictoires, trop empreintes encore des traces tenaces du mépris patriarcal du corps, cette ambivalence semble refléter moins le refus d'un nouvel ancrage corporel que la fragilité du sujet qui, pris dans son abjecte panique d'animal traqué (P, p. 105), ne sait pas comment refaire son destin de mâle.

Ce sont les dimensions de cette fragilité comme l'impérieuse nécessité d'une redéfinition corporelle que traduisent les autres images du corps masculin dans les Masques et le Passager. Outre des allusions isoléés à diverses parties du corps que nous ne retiendrons pas aux fins de cette analyse, chaque livre offre des configurations corporelles différentes en rapport avec la thématique générale qui l'informe. C'est donc le moment d'examiner ces œuvres séparément, ce qui permettra de soulever la question d'une éventuelle évolution d'un livre à l'autre.

À l'instar de son titre, double image de l'identité apparente et de la réalité profonde ${ }^{31}$, masque et face, costume et peau (M, p. 21), les Masques mettent surtout en relief l'enveloppe corporelle, normalement garante de l'intégrité du sujet bien dans sa peau ${ }^{32}$. Mais si le livre foisonne d'images et de métaphores de la peau, celle-ci paraît invariablement fragile et douloureuse, sujette à la détérioration. Le personnage masculin se gratte avec [ses] ongles rongés (M, p. 53). Sa peau est toujours en sueur, sa chemise mouillée (M, p. 13), son jeans lui colla[nt] aux cuisses (M, p. 23), autant d'échos de l'image

30 Voir à ce sujet $\mathrm{S}$. Bertho, "Le moi et la bête: le bestiaire d'Henri Michaux", l'Homme et l'animal, ouvrage collectif sous la direction de S. Briosi et J. Lintvelt, CRIN 19 (1988), p. 120-125.

31 J. Chevalier et A. Gheerbrant, op. cit., p. 617.

32 D. Anzieu, loc. cit., p. 204. 
saisissante et maintes fois répétée de l'enfant qui attend la punition dans la chaleur mouillée, puante et abjecte de son lit (M, p. 36), déjà atteint dans les pelures sensibles de son âme (M, p. 36).

Tels les vêtements de l'enfant noyé, ce paquet de chiffons rouge et blanc (M, p. 32) réduit en varechs fumants et immondes (M, p. 31), et les couvertures [que] l'agonisant tire sun son corps qui se refroidit (M, p. 101), la peau ne parvient à protéger le sujet ni des attaques de l'extérieur, par les grandes mains sèches (M, p. 67) du père qui le bat, ni surtout des angoisses d'éclatement ou de "vidange». Se sentant littéralement tomber en jus (M, p. 13), mot qui désigne aussi bien l'urine (dans mes couvertes, je me tourne et me retourne, viraille dans mon jus sale [M, p. 53]) que. le sperme, jus essentiel (M, p. 81), le personnage masculin n'arrive pas à contenir l'ectoplasme [...] flottant à l'intérieur (M, p. 21) de lui. Dans sa peau douloureuse, il a souvent le cœur qui lève (M, p. 64), ou bien l'impression inverse d'être aspiré dans le noir clapotant hideusement au fond de [lui]-même (M, p. 89). Même l'écriture se présente comme une explosion ambiguë:

ça n'aurait pris [...] qu'un petit sursaut de sa volonté pour que tout lui échappe [...] et que par les vannes ouvertes les mots jaillissent de lui comme une trombe, que sa création s'écoule de lui telle une éjaculation qu'on ne peut plus retenir. (M, p. 27-28)

Dans le contexte d'un tel moi-peau ${ }^{33}$ fragile et perméable, il n'est pas étonnant que la redéfinition du corps passe par la descente intérieure dans les tréfonds liquides du sujet. Le protagoniste des Masques entreprend ainsi une sorte de noyade intérieure (M, p. 114) positive qui permet la récupération symbolique de la figure de l'enfant noyé, son fils en l'occurrence, qui hante l'intrigue du livre. Descendant vers cette rue de son enfance (M, p. 43), au plus profond de lui où depuis trente ans une forme obscure tournait en rond comme un enfant qui a perdu son chemin (M, p. 112), le personnage passe par une transformation plus terrible et plus irrémédiable que la simple mort [...] d'où il ressortirait [...], sa blessure enfin cautérisée, capable désormais d'aller plus haut et de vivre vraiment debout (M, p. 112). Réaffirmation de cette stérile présomption du sujet masculin debout et seul sur la surface d'un globe réduit à l'état d'un objet ${ }^{34}$, ou découverte d'une nouvelle verticalité masculine?

S'ajoutant à l'espoir d'une blessure cautérisée, les images de la peau se transforment en fait au cours du livre pour revaloriser, - quoique de manière encore ambiguë, l'intégrité du sujet masculin. Lieu d'abord d'éclatements dévalorisants, tant sur le plan affectif

33 Ibid.

34 Patricia Smart, op. cit., p. 175. 
(tout était en train de péter dans mon cœur [...] ma vie entière s'en allait déchirée en lambeaux ça me partait de sur le corps comme peau de lépreux [M, p. 95]), que sexuel (j'avais le sperme qui me montait pour ainsi dire à la tête et qui me sortait par les pores [M, p. 86]), la peau finit par céder à une sorte d'irrigation douce-amère grâce à laquelle le sujet réintègre son corps et fait revivre son masque:

je marchais en suffoquant, hoquetant misérable, soudain gonflé de trop gros sanglots qui sortaient mal de ce visage habitué à porter son masque qui étaient expulsés en somme de tout mon corps et toute mon âme [...] toute la douleur que javais depuis si longtemps en moi et qui avait enfin pris forme et liquide me sortait par la face et ruisselait dans ma barbe [...] drainé de toute force et de toute velléité de résistance, faible comme un nouveau-né, il fallait que je m'amollisse suffisamment pour prendre la forme que le sort voulait me donner. (M, p. 188-189)

Portrait poignant d'une affectivité blessée pleinement assumée, ce passage n'offre pourtant aucune véritable réintégration du corps sexuel, mis pour ainsi dire en suspens, dans le corps amorphe du nouveau-né.

C'est précisément cette question du corps sexuel qui traverse le Passager que l'on peut considérer ainsi comme l'approfondissement, pénible mais tangible, du livre précédent. Fidèle aussi à son titre mais plus violent que les Masques, le dernier livre de La Rocque privilégie les images du corps en mouvement. Mouvement surtout des mains qui bougent pour frapper et des pieds qui marchent à la recherche d'un soulagement, vengeance ou tendresse compensatoire. Telle l'image de l'enfant attendant anxieux dans son lit mouillé qui alimente toute l'imagerie corporelle des Masques, c'est la scène de la mort du canari aux mains du jeune héros du Passager qui fournit ici la clé des configurations corporelles:

dans sa main l'oiseau n'était qu'un frémissement tiède et doux, et l'enfant serrà il serra de toute sa force jusqu'à ce qu'il eût mal dans ses jointures et il ferma les yeux, pris d'un énorme tremblement qui le secouait tout entier. $\left(\mathbf{P}, \mathbf{p}_{\text {. }}\right.$ 12)

Survenu à la suite d'un incident où le jeune Bernard a été frappé par les mains osseuses, dures et froides de son père $(\mathbf{P}, \mathbf{p} .11)$, ce passage rapproche les mains et le sexe, par l'ambivalence du mot oiseau et l'évocation négative de la masturbation, dans un rapport de violence qui dominera l'imagerie corporelle du texte entier.

Organe aux fonctions naturelles variées et doté d'acceptions syñboliques diverses ${ }^{35}$, la main est essentiellement réduite dans le

35 Pierre Guiraud, op. cit., p. 51. 
Passager à deux valeurs, utilisée parfois comme indice d'amitié (la main de Paul serrait son bras et le soutenait $[\mathbf{P}, \mathbf{p} .76])$, mais surtout comme agent de domination. Le héros se sent emprisonné par des mains qui le saisissent un peu partout, on le ceinturait, on le tirait, on le poussait, certains en profitaient pour le pincer ou lui donner des coups (P, p. 58). Craignant, enfant, les mains dures de son père (P, p. 149), leur geste brutal (P, p. 149), il est hanté, adulte, par l'image intolérable de Guilbert lui faisant face et le narguant et le giflant à la volée $(\mathbf{P}, \mathbf{p} .81)$ ou saisi par la peur plaquée comme une main froide entre les omoplates (P, p. 147), par une main qui lui écrasait les tripes $(\mathbf{P}$, p. 113). Ses propres mains sont également violentes: il serrait aussi ses mains entre ses genoux, comme s'il avait peur qu'elles ne lui échappent comme des bêtes malfaisantes (P, p. 137); il sentait pendre hideusement ses mains au bout de ses bras [...] ces morceaux d'os et de chair qu'il aurait voulu pouvoir amputer, sachant bien que désormais [...] elles ne cesseraient plus jamais d'être les mains qui avaient tué Liliane (P, p. 140-141).

Fermées en poings, les mains entrent alors dans tout un réseau d'associations corporelles qui les relie de manière plus explicite à une vision patriarcale, violente, de la sexualité. Figure presque obsessionnelle du malaise physique ou affectif du héros (les poings crispés au fond de ses poches [P, p. 104]; une terrible crampe qui lui ravageait le poignet $[\mathbf{P}, \mathrm{p} .111]$; il se voyait tout poigné dans ce vaste brassage de peaux en sueur [P, p. 28]), le poing se transforme en poignard (il toucha du bout des doigts le manche du poignard, sous son coupevent $[P, p .102]$ ou en pénis (il se contentait de tripoter le manche de son poignard comme un enfant timide qui joue avec son pénis quand on lui parle [P, p. 116]) ou bien s'associe, par métonymie, à l'acte sexuel (il avait envie de se passer un poignet de qualité $[\mathbf{P}, \mathbf{p} .92]$; la main poignant subreptice le fruit mollasse [P, p. 88]). Enfin, la clé de ce réseau d'associations dévalorisantes est fournie par la description du pénis paternel qui déclenche le désarroi du héros à la fin du livre: il était bandé gros et dur il se maniait branlait en ricanant son instrument gigantesque gland violacé comme un champignon vénéneux émergeant de son poing amanite immonde ( $\mathrm{P}, \mathrm{p} .199)$.

Cette même image violente du phallus patriarcal guette la quête de verticalité du héros, toujours planté droit $(\mathbf{P}$, p. 102) quelque part à attendre ou se voyant debout à l'intérieur de sa tête $(\mathbf{P}, \mathrm{p} .102)$. Alternant avec ces images d'immobilité, l'errance à pied, presque toujours dans la boue ou des flaques d'eau, traduit une tentative de renouer avec la base $d u$ corps $^{36}$, d'en assurer l'occupation ${ }^{37}$. Reliée

36 Pierre Guiraud, ibid., p. 53.

37 Le pied symboliserait moins la possession que l'occupation avec l'intention de défendre (Ibid.). 
au thème de la maison du grand-oncle Émilien, seul homme à avoir traité le père du héros de trou d'cul $(\mathbf{P}$, p. 24), cette tentative est pourtant destinée à échouer. Si, enfant, le héros gardait un pied sur la planche du fossé, sentant derrière lui [...] cette maison où il allait ' pouvoir dans quelques instants pénétrer comme chez lui, sans même cogner (P, p. 18. Nous soulignons), adulte, il ne peut que répéter le geste de son père qui avait dû frapper à grands coups de poing dans la porte (P, p. 174).

Contrairement à son père, pourtant, le héros du Passager finit par arrêter son geste. Il garde néanmoins le poing meurtri (P, p. 175), repartant dans l'errance, sans savoir où il allait, n'allant nulle part en réalité, rentrant en lui-même pour y disparaître, vacillant sur ses jambes molles, avec cette tête trop lourde qui l'attirait puissamment vers la terre (P, p. 176). Tout comme il est conscient, après avoir tué, en fantasme, la femme avec qui il vit, que plus jamais il ne pourrait marcher dans ses propres traces (P, p. 145), le héros semble reconnaître ici l'impossibilité d'une verticalité qui n'impliquerait pas un nouvel équilibre du corps et de la tête. Seulement, épuisé et démuni, il ne parvient pas à définir cet équilibre de manière positive. Par sa forme comme par sa logique, la tentative de suicide du héros emprisonné, littéralement et symboliquement, à la fin du livre reprend ainsi l'image de l'oiseau étouffé: il se lâcha dans le vide. Ses pieds heurtèrent les barreaux d'acier [...] tandis qu'une douleur foudroyante, absolument inconcevable, le poignait dans le cou, lui tordait la nuque et lui écrasait la gorge (P, p. 204).

Pénible itinéraire, donc, que ces images douloureuses, mais courageuses, du corps masculin dans l'œuvre de La Rocque. Si, dans les Masques, le personnage masculin parvient à -renouer avec certains aspects du féminin, comme en témoignent la thématique de la descente dans l'eau et la revalorisation de l'affectif, il ne parvient pas pour autant à réintégrer et à réinventer son corps sexuel. Le modèle patriarcal de l'identité masculine reste encore intact. Le protagoniste retrouve l'enfant blessé au fond de lui-même, sans pour autant pouvoir briser le cycle. Son enfant à lui se noie, à la suite d'un incident où, tel son père brutal, il l'aurait frappé à son tour, de sa main (M, p. 150151). Dans le Passager, pourtant, c'est justemént cette brutalité du modèle patriarcal, comme le tenace obstacle qu'il pose à la réinvention du corps sexuel masculin, qui sont enfin confrontés et déconstruits.

Plus qu'une écriture de transition, tiraillée entre l'impossible «identification paternelle" et la difficile revalorisation du féminin, pour reprendre la perspective de Nepveu et de Smart, Gilbert La Rocque nous laisse en fin de compte, nous semble-t-il; l'image d'une fragile renaissance du corps masculin. Certes, la revalorisation de l'affectivité douloureuse du sujet et la déconstruction du modèle patriarcal de la sexualité, du moins en ce qui concerne le corps de 
l'homme, aboutissent, d'une manière ou d'une autre, à la mort symbolique du sujet masculin. Dans les deux-romans pourtant, cette mort est relativisée, présentée comme une étape intermédiaire. Se donnant des années de rumination pour consommer entièrement cette forme spéciale de solitude morale (M, p. 190), le protagoniste des Masques se réjouit quand même du bel été (M, p. 191) à venir. Malgré son air d'un cadavre qu'on mène au cimetière (P, p. 212), le héros du Passager ne rentre pas moins chez la femme qu'il croyait avoir tuée. Il est donc permis d'espérer que le suicide raté du protagoniste symbolise non pas l'éclatement du sujet, mais plutôt la table rase à partir de laquelle il lui faudrait redéfinir la vie incertaine avec l'Autre. 